\title{
A UNIFYING APPROACH FOR CERTAIN CLASS OF MAXIMAL FUNCTIONS
}

\author{
AHMAD AL-SALMAN
}

Received 16 January 2006; Revised 12 April 2006; Accepted 13 April 2006

We establish $L^{p}$ estimates for certain class of maximal functions with kernels in $L^{q}\left(\mathbf{S}^{n-1}\right)$. As a consequence of such $L^{p}$ estimates, we obtain the $L^{p}$ boundedness of our maximal functions when their kernels are in $L(\log L)^{1 / 2}\left(\mathbf{S}^{n-1}\right)$ or in the block space $B_{q}^{0,-1 / 2}\left(\mathbf{S}^{n-1}\right)$, $q>1$. Several applications of our results are also presented.

Copyright (c) 2006 Ahmad Al-Salman. This is an open access article distributed under the Creative Commons Attribution License, which permits unrestricted use, distribution, and reproduction in any medium, provided the original work is properly cited.

\section{Introduction and statement of results}

Let $\mathbb{R}^{n}, n \geq 2$, be the $n$-dimensional Euclidean space and let $\mathbf{S}^{n-1}$ be the unit sphere in $\mathbb{R}^{n}$ equipped with the normalized Lebesgue measure $d \sigma$. For nonzero $y \in \mathbb{R}^{n}$, we will let $y^{\prime}=|y|^{-1} y$. Let $\Omega$ be an integrable function on $\mathbf{S}^{n-1}$ that is homogeneous of degree zero on $\mathbb{R}^{n}$ and satisfies the cancelation property

$$
\int_{S^{n-1}} \Omega\left(y^{\prime}\right) d \sigma\left(y^{\prime}\right)=0
$$

Consider the maximal function $M_{\Omega}$,

$$
M_{\Omega}(f)(x)=\left.\sup _{h \in U}\left|\int_{\mathbb{R}^{n}} f(x-y)\right| y\right|^{-n} h(|y|) \Omega\left(y^{\prime}\right) d y \mid,
$$

where $U$ is the class of all $h \in L^{2}\left(\mathbb{R}_{+}, r^{-1} d r\right)$ with $\|h\|_{L^{2}\left(\mathbb{R}_{+}, r^{-1} d r\right)} \leq 1$.

The operator $M_{\Omega}$ was introduced by Chen and Lin [7]. They showed that $M_{\Omega}$ is bounded on $L^{p}\left(\mathbb{R}^{n}\right)$ for all $p>2 n /(2 n-1)$ provided that $\Omega \in \mathscr{C}\left(\mathbf{S}^{n-1}\right)$. Recently, we have been able to show that the $L^{p}\left(\mathbb{R}^{n}\right)$ boundedness of $M_{\Omega}$ still holds for all $p \geq 2$ if the condition $\Omega \in \mathscr{C}\left(\mathbf{S}^{n-1}\right)$ is replaced by the more natural and weaker condition $\Omega \in$ $L(\log L)^{1 / 2}\left(\mathbf{S}^{n-1}\right)$ [2]. Moreover, we showed that if the condition $\Omega \in L(\log L)^{1 / 2}\left(\mathbf{S}^{n-1}\right)$ is replaced by any condition in the form $\Omega \in L(\log L)^{r}\left(\mathbf{S}^{n-1}\right)$ for some $r<1 / 2$, then $M_{\Omega}$ might fail to be bounded on $L^{2}$. 
On the other hand, when $\Omega$ lies in $B_{s}^{0,-1 / 2}\left(\mathbf{S}^{n-1}\right), s>1$, which is a special class of block spaces $B_{q}^{\kappa, v}\left(\mathbf{S}^{n-1}\right)$ (see Section 5 for the definition), we were able to show that $M_{\Omega}$ is bounded on $L^{p}$ for all $p \geq 2$ [3]. Moreover, we showed that the condition $\Omega \in$ $B_{s}^{0,-1 / 2}\left(\mathbf{S}^{n-1}\right), s>1$ is nearly optimal in the sense that the exponent $-1 / 2$ cannot be replaced by any smaller number for the $L^{2}$ boundedness of $M_{\Omega}$ to hold. We remark here that block spaces have been introduced by Jiang and Lu to improve previously obtained $L^{p}$ boundedness results for singular integrals [7]. It should be noted here that the relation between the spaces $B_{s}^{0,-1 / 2}\left(\mathbf{S}^{n-1}\right)$ and $L(\log L)^{1 / 2}\left(\mathbf{S}^{n-1}\right)$ is unknown.

However, it is known that $L^{q}\left(\mathbf{S}^{n-1}\right)$ is properly contained in $L(\log L)^{1 / 2}\left(\mathbf{S}^{n-1}\right) \cap$ $B_{s}^{0,-1 / 2}\left(\mathbf{S}^{n-1}\right)$ for all $q, s>1$. Moreover, it is not hard to see that every $\Omega$ in $L(\log L)^{1 / 2}\left(\mathbf{S}^{n-1}\right) \cup B_{s}^{0,-1 / 2}\left(\mathbf{S}^{n-1}\right)$ can be written as an infinite sum of functions in $L^{q}\left(\mathbf{S}^{n-1}\right)$. This gives rise to the question whether the results pertaining the $L^{p}$ boundedness of $M_{\Omega}$ in $[2,3]$ can be obtained via certain corresponding $L^{p}$ estimates with kernels in $L^{q}\left(\mathbf{S}^{n-1}\right)$. It is one of our main goals in this paper to consider such problem. It should be pointed out here that a positive solution for this problem will not only make life easier when dealing with kernels in $L(\log L)^{1 / 2}\left(\mathbf{S}^{n-1}\right)$ or $B_{s}^{0,-1 / 2}\left(\mathbf{S}^{n-1}\right)$, but also will pave the way for extending several results that are known when kernels are in $L^{q}\left(\mathbf{S}^{n-1}\right)$.

Our work in this paper will be mainly concerned with the following general class of maximal functions:

$$
M_{\Omega, P}(f)(x)=\left.\sup _{h \in U}\left|\int_{\mathbb{R}^{n}} e^{i P(y)} f(x-y)\right| y\right|^{-n} h(|y|) \Omega\left(y^{\prime}\right) d y \mid,
$$

where $P: \mathbb{R}^{n} \rightarrow \mathbb{R}$ is a real-valued polynomial.

Clearly, if $P(y)=0$, then $M_{\Omega, P}=M_{\Omega}$. For the significance of considering integral operators with oscillating kernels, we refer the readers to consult $[1,4,11,16,19,22-24]$, among others.

Our result concerning $L^{p}$ estimates with kernels in $L^{q}\left(\mathbf{S}^{n-1}\right)$ is the following theorem.

Theorem 1.1. Let $\Omega \in L^{q}\left(\mathbf{S}^{n-1}\right), q>1$, be a homogeneous function of degree zero on $\mathbb{R}^{n}$ with $\|\Omega\|_{1} \leq 1$. Let $P: \mathbb{R}^{n} \rightarrow \mathbb{R}$ be a real-valued polynomial of degree d. Let $M_{\Omega, P}$ be given by (1.3). Then

$$
\left\|M_{\Omega, P}(f)\right\|_{p} \leq\left\{1+\log ^{1 / 2}\left(e+\|\Omega\|_{q}\right)\right\} C_{p, q}\|f\|_{p}
$$

for all $p \geq 2$, where $C_{p, q}=\left(2^{1 / q^{\prime}} /\left(2^{1 / q^{\prime}}-1\right)\right) C_{p}$. Here $1 / q^{\prime}=1-1 / q$ and $C_{p}$ is a constant that may depend on the degree of the polynomial $P$ but it is independent of the function $\Omega$, the index $q$, and the coefficients of the polynomial $P$.

We remark here that the constant $C_{p, q}$ in Theorem 1.1 satisfies $C_{p, q} \rightarrow \infty$ as $q \rightarrow 1^{+}$. That is, the constant $C_{p, q}$ diverges when $q$ tends to 1 . This behavior of $C_{p, q}$ is natural since, by [2, Theorem $\mathrm{B}(\mathrm{b})]$, the special operator $M_{\Omega}=M_{\Omega, 0}$ is not bounded on $L^{2}$ if the function $\Omega$ is assumed to satisfy only the sole condition that $\Omega \in L^{1}\left(\mathbf{S}^{n-1}\right)$ (i.e., $q=1$ ).

By a suitable decomposition of the function $\Omega$ and an application of Theorem 1.1, we prove the following theorem which is a proper extension of the corresponding result in $[2]$. 
Theorem 1.2. Suppose that $\Omega \in L\left(\log ^{+} L\right)^{1 / 2}\left(\mathbf{S}^{n-1}\right)$ satisfying $(1.1)$. Let $P: \mathbb{R}^{n} \rightarrow \mathbb{R}$ be a real-valued polynomial. Then $M_{\Omega, P}$ is bounded on $L^{p}\left(\mathbb{R}^{n}\right)$ for all $p \geq 2$ with $L^{p}$ bounds independent of the coefficients of the polynomial P.

We should point out here that an alternative proof of Theorem 1.2 can be obtained by observing that $C_{p, q} \approx C_{p} /(q-1)$, where $C_{p, q}$ is the constant in Theorem 1.1, and then using a Yano-type extrapolation technique [27].

By another suitable application of Theorem 1.1, we will prove the following extension of [3, Theorem 1.2].

Theorem 1.3. Suppose that $\Omega \in B_{q}^{0,-1 / 2}\left(\mathbf{S}^{n-1}\right), q>1$, satisfying (1.1). Let $P: \mathbb{R}^{n} \rightarrow \mathbb{R}$ be a real-valued polynomial. Then $M_{\Omega, P}$ is bounded on $L^{p}\left(\mathbb{R}^{n}\right)$ for all $p \geq 2$ with $L^{p}$ bounds independent of the coefficients of the polynomial $P$.

As an immediate consequence of Theorem 1.1 and the observation that

$$
\left|T_{\Omega, P, h}(f)(x)\right| \leq\|h\|_{L^{2}\left(\mathbb{R}_{+}, r^{-1} d r\right)} \mathcal{M}_{\Omega, P}(f)(x),
$$

we obtain the following result concerning oscillatory singular integrals.

Theorem 1.4. Let $\Omega \in L^{q}\left(\mathbf{S}^{n-1}\right), q>1$, be a homogeneous function of degree zero on $\mathbb{R}^{n}$ with $\|\Omega\|_{1} \leq 1$. Let $P: \mathbb{R}^{n} \rightarrow \mathbb{R}$ be a real-valued polynomial of degree $d$ and let $h \in$ $L^{2}\left(\mathbb{R}_{+}, r^{-1} d r\right)$. Then the oscillatory singular integral operator $M_{\Omega, P}$;

$$
T_{\Omega, P, h}(f)(x)=p \cdot v \int_{\mathbb{R}^{n}} e^{i P(y)} f(x-y)|y|^{-n} h(|y|) \Omega\left(y^{\prime}\right) d y
$$

satisfies

$$
\left\|T_{\Omega, P, h}(f)\right\|_{p} \leq\left\{1+\log ^{1 / 2}\left(e+\|\Omega\|_{q}\right)\right\}\|h\|_{L^{2}\left(\mathbb{R}_{+}, r^{-1} d r\right)} C_{p, q}\|f\|_{p}
$$

for all $p \geq 2$, where $C_{p, q}=\left(2^{1 / q^{\prime}} /\left(2^{1 / q^{\prime}}-1\right)\right) C_{p}$. Here $1 / q^{\prime}=1-1 / q$ and $C_{p}$ is a constant that may depend on the degree of the polynomial $P$ but it is independent of the function $\Omega$, the index $q$, and the coefficients of the polynomial $P$.

By Theorem 1.4, we obtain the following two results.

Corollary 1.5. Let $\Omega \in L(\log L)^{1 / 2}\left(\mathbf{S}^{n-1}\right)$ be a homogeneous function of degree zero on $\mathbb{R}^{n}$ and satisfies (1.1). Let $P: \mathbb{R}^{n} \rightarrow \mathbb{R}$ be a real-valued polynomial of degree $d$ and let $h \in$ $L^{2}\left(\mathbb{R}_{+}, r^{-1} d r\right)$. Then the oscillatory singular integral operator $M_{\Omega, P}$;

$$
T_{\Omega, P, h}(f)(x)=p \cdot v \int_{\mathbb{R}^{n}} e^{i P(y)} f(x-y)|y|^{-n} h(|y|) \Omega\left(y^{\prime}\right) d y
$$

is bounded on $L^{p}$ for all $p \geq 2$ with $L^{p}$ bounds that may depend on the degree of the polynomial $P$ but they are independent of the coefficients of the polynomial P.

Corollary 1.6. Let $\Omega \in B_{q}^{0,-1 / 2}\left(\mathbf{S}^{n-1}\right), s>1$, be a homogeneous function of degree zero on $\mathbb{R}^{n}$ and satisfies (1.1). Let $P: \mathbb{R}^{n} \rightarrow \mathbb{R}$ be a real-valued polynomial of degree $d$ and let 
4 A unifying approach for certain class of maximal functions

$h \in L^{2}\left(\mathbb{R}_{+}, r^{-1} d r\right)$. Then the oscillatory singular integral operator $M_{\Omega, P}$;

$$
T_{\Omega, P, h}(f)(x)=p \cdot v \int_{\mathbb{R}^{n}} e^{i P(y)} f(x-y)|y|^{-n} h(|y|) \Omega\left(y^{\prime}\right) d y
$$

is bounded on $L^{p}$ for all $p \geq 2$ with $L^{p}$ bounds that may depend on the degree of the polynomial $P$ but they are independent of the coefficients of the polynomial $P$.

Further applications of the results stated above will be presented in Section 6.

Throughout this paper, the letter $C$ will stand for a constant that may vary at each occurrence, but it is independent of the essential variables.

\section{Preliminary estimates}

We start by recalling the following result in [10].

Lemma 2.1 (see $[10])$. Let $\mathscr{P}=\left(P_{1}, \ldots, P_{d}\right)$ be a polynomial mapping from $\mathbb{R}^{n}$ into $\mathbb{R}^{d}$. Suppose that $\Omega \in L^{1}\left(\mathbf{S}^{n-1}\right)$ and

$$
M_{\Omega, \mathscr{P}} f(x)=\sup _{j \in \mathbb{Z}} \int_{2^{j} \leq|y|<2^{j+1}}|f(x-\mathscr{P}(y))||y|^{-n}\left|\Omega\left(y^{\prime}\right)\right| d y .
$$

Then for $1<p \leq \infty$, there exist a constant $C_{p}>0$ independent of $\Omega$ and the coefficients of $P_{1}, \ldots, P_{d}$ such that

$$
\left\|M_{\Omega, \mathscr{P}} f\right\|_{p} \leq C_{p}\|\Omega\|_{L^{1}\left(\mathbf{S}^{n-1}\right)}\|f\|_{p}
$$

for every $f \in L^{p}\left(\mathbb{R}^{d}\right)$.

Lemma 2.2 (van der Corput [26]). Suppose $\phi$ is real valued and smooth in $(a, b)$, and that $\left|\phi^{(k)}(t)\right| \geq 1$ for all $t \in(a, b)$. Then the inequality

$$
\left|\int_{a}^{b} e^{-i \lambda \phi(t)} \psi(t) d t\right| \leq C_{k}|\lambda|^{-1 / k}
$$

holds when

(i) $k \geq 2$, or

(ii) $k=1$ and $\phi^{\prime}$ is monotonic.

The bound $C_{k}$ is independent of $a, b, \phi$, and $\lambda$.

Lemma 2.3. Let $\Omega \in L^{q}\left(\mathbf{S}^{n-1}\right), q>1$, be a homogeneous function of degree zero on $\mathbb{R}^{n}$ with $\|\Omega\|_{1} \leq 1$. Let $P(x)=\sum_{|\alpha| \leq d} a_{\alpha} x^{\alpha}$ be a real-valued polynomial of degree $d>1$ such that $|x|^{d}$ is not one of its terms. For $k \in \mathbb{Z}$, let $E_{k, \Omega}:\left[1, \log \left(e+\|\Omega\|_{q}\right)\right] \times P\left(\mathbf{S}^{n-1}\right) \times \mathbb{R} \rightarrow \mathbb{C}$ and let $\mathbf{J}_{k, \Omega}: \mathbb{R}^{n} \rightarrow \mathbb{R}$ be given by

$$
\begin{gathered}
E_{k, \Omega}\left(r, P\left(y^{\prime}\right), s\right)=e^{-i\left[P\left(2^{-(k+1) \log \left(e+\|\Omega\| \|_{q}\right)} r y^{\prime}\right)+2^{-(k+1) \log \left(e+\|\Omega\| \|_{q}\right)} s r\right]}, \\
\mathbf{J}_{k, \Omega}(\xi)=\int_{1}^{2^{2 \log (e+\|\Omega\| q)}}\left|\int_{\mathbf{S}^{n-1}} \Omega\left(y^{\prime}\right) E_{k, \Omega}\left(r, P\left(y^{\prime}\right), \xi \cdot y^{\prime}\right) d \sigma\left(y^{\prime}\right)\right|^{2} \frac{d}{r} r .
\end{gathered}
$$


Then, $\mathbf{J}_{k, \Omega}$ satisfies

$$
\sup _{\xi \in \mathbb{R}^{n}} \mathbf{J}_{k, \Omega}(\xi) \leq 2^{(k+1) / 4 q^{\prime}} \log \left(e+\|\Omega\|_{q}\right)\left\{\sum_{|\alpha|=d}\left|a_{\alpha}\right|\right\}^{-\varepsilon / q^{\prime}} C
$$

for some $0<\varepsilon<1$, where $C$ is a constant that may depend on the degree of the polynomial $P$ but it is independent of the function $\Omega$, the index $q$, and the coefficients of the polynomial $P$. Proof of Lemma 2.3. First, we notice the following:

$$
\begin{gathered}
\mathbf{J}_{k, \Omega}(\xi) \leq \log \left(e+\|\Omega\|_{q}\right), \\
\left(\mathbf{J}_{k, \Omega}(\xi)\right)^{q^{\prime}} \leq\|\Omega\|_{q}^{2 q^{\prime}} \iint_{S^{n-1}} \mid \int_{1}^{2^{2 \log \left(e+\|\Omega\|_{q}\right)}} \\
E_{k, \Omega}\left(r, P\left(y^{\prime}\right), \xi \cdot y^{\prime}\right) \\
\times\left.\overline{E_{k, \Omega}\left(r, P\left(z^{\prime}\right), \xi \cdot z^{\prime}\right)} \frac{d r}{r}\right|^{q^{\prime}} d \sigma\left(y^{\prime}\right) d \sigma\left(z^{\prime}\right) .
\end{gathered}
$$

Next, notice that

$$
\begin{aligned}
& P\left(2^{-\gamma_{k, \Omega}} r y^{\prime}\right)+2^{-\gamma_{k, \Omega}}\left(\xi \cdot y^{\prime}\right) r-P\left(2^{-\gamma_{k, \Omega}} r z^{\prime}\right)+2^{-\gamma_{k, \Omega}}\left(\xi \cdot z^{\prime}\right) r \\
& \quad=2^{-\gamma_{k, \Omega} d} r^{d}\left\{\sum_{|\alpha|=d} a_{\alpha} y^{\prime \alpha}-\sum_{|\alpha|=d} a_{\alpha} z^{\prime \alpha}\right\}+2^{-\gamma_{k, \Omega}} \xi \cdot\left(y^{\prime}-z^{\prime}\right) r+H_{k}\left(r, y^{\prime}, z^{\prime}, \xi\right)
\end{aligned}
$$

with $\left(d^{d} / d r^{d}\right) H_{k}=0$, where $\gamma_{k, \Omega}=(k+1) \log \left(e+\|\Omega\|_{q}\right)$. Thus, by Lemma 2.2, we have

$$
\left|\int_{1}^{2^{2 \log \left(e+\|\Omega\|_{q}\right)}} E_{k, \Omega}\left(r, P\left(y^{\prime}\right), \xi \cdot y^{\prime}\right) \overline{E_{k, \Omega}\left(r, P\left(z^{\prime}\right), \xi \cdot z^{\prime}\right)} \frac{d r}{r}\right| \leq\left|2^{-d \gamma_{k, \Omega}}\left\{P\left(y^{\prime}\right)-P\left(z^{\prime}\right)\right\}\right|^{-1 / d} .
$$

Now, by (2.9) and the inequality

$$
\left|\int_{1}^{2^{2 \log \left(e+\|\Omega\|_{q}\right)}} E_{k, \Omega}\left(r, P\left(y^{\prime}\right), \xi \cdot y^{\prime}\right) \overline{E_{k, \Omega}\left(r, P\left(z^{\prime}\right), \xi \cdot z^{\prime}\right)} \frac{d r}{r}\right| \leq C \log \left(e+\|\Omega\|_{q}\right),
$$

we obtain

$$
\begin{aligned}
& \left|\int_{1}^{2^{2 \log (e+\|\Omega\|} \|_{q}} E_{k, \Omega}\left(r, P\left(y^{\prime}\right), \xi \cdot y^{\prime}\right) \frac{E_{k, \Omega}\left(r, P\left(z^{\prime}\right), \xi \cdot z^{\prime}\right)}{r} \frac{d r}{r}\right| \\
& \quad \leq\left|2^{-d \gamma_{k, \Omega}}\left\{P\left(y^{\prime}\right)-P\left(z^{\prime}\right)\right\}\right|^{-1 / 4 d q^{\prime}} C\left\{\log \left(e+\|\Omega\|_{q}\right)\right\}^{1-1 / 4 q^{\prime}} .
\end{aligned}
$$

Therefore, by (2.7), (2.11), and [12, (3.11)], we obtain

$$
\mathbf{J}_{k, \Omega}(\xi) \leq 2^{\gamma_{k, \Omega / 4 q^{\prime}}}\|\Omega\|_{q}^{2 q^{\prime}} C\left\{\log \left(e+\|\Omega\|_{q}\right)\right\}^{1-1 / 4 q^{\prime}} .
$$


6 A unifying approach for certain class of maximal functions

Hence, by (2.6) and (2.12), we get

$$
\begin{aligned}
\mathbf{J}_{k, \Omega}(\xi) & \leq 2^{\gamma_{k, \Omega} / 4 \log \left(e+\|\Omega\|_{q}\right) q^{\prime}}\|\Omega\|_{q}^{2 / \log \left(e+\|\Omega\|_{q}\right)} \log \left(e+\|\Omega\|_{q}\right) \\
& \leq 2^{(k+1) / 4 q^{\prime}} \log \left(e+\|\Omega\|_{q}\right) C .
\end{aligned}
$$

This completes the proof.

Now, we will need the following lemma.

Lemma 2.4. Let $\Omega \in L^{q}\left(\mathbf{S}^{n-1}\right), q>1$, be a homogeneous function of degree zero on $\mathbb{R}^{n}$ with $\|\Omega\|_{1} \leq 1$. Then

$$
\left\|M_{\Omega}(f)\right\|_{p} \leq \log ^{1 / 2}\left(e+\|\Omega\|_{q}\right)\left\{\frac{2^{1 / q^{\prime}}}{2^{1 / q^{\prime}}-1}\right\} C_{p}\|f\|_{p}
$$

for all $p \geq 2$ with constants $C_{p}$ independent of the function $\Omega$ and the index $q$.

We remark here that since $L^{q}\left(\mathbf{S}^{n-1}\right) \subset L \log ^{1 / 2} L$, it follows from [2, Theorem B(a)] that $\left\|M_{\Omega}\right\|_{p} \leq\|\Omega\|_{q} C_{p}$ for all $p \geq 2$. But, clearly the constant $\left\{1+\log ^{1 / 2}\left(e+\|\Omega\|_{q}\right)\right\}$ in $(2.14)$ is sharper than the constant $\|\Omega\|_{q}$ that can be deduced from [2, Theorem $\left.\mathrm{B}(\mathrm{a})\right]$. However, the former constant can be obtained by following a similar argument as in the proof of Theorem B(a) in [2] and keeping track of certain constants. For completeness, we, below, present the main ideas of the proof.

Proof of Lemma 2.4. Choose a collection of $\mathscr{C}^{\infty}$ functions $\left\{\omega_{k}\right\}_{k \in \mathbb{Z}}$ on $(0, \infty)$ with the properties $\sup \left(\omega_{k}\right) \subseteq\left[2^{-\log \left(e+\|\Omega\|_{q}\right)(k+1)}, 2^{-\log \left(e+\|\Omega\|_{q}\right)(k-1)}\right], 0 \leq \omega_{k} \leq 1, \sum_{k \in \mathbb{Z}} \omega_{k}(u)=1$, $\left|\left(d^{s} \omega_{k} / d u^{s}\right)(u)\right| \leq C_{s} u^{-s}$, where $C_{s}$ is a constant independent of $\log \left(e+\|\Omega\|_{q}\right)$. For $k \in \mathbb{Z}$, let $G_{k}$ be the operator defined by $\left(G_{k}(f) \hat{)}(\xi)=\omega_{k}(|\xi|) \hat{f}(\xi)\right.$. Let

$$
E_{j}(f)(x)=\left(\sum_{k \in \mathbb{Z}} \int_{1}^{2^{2 \log \left(e+\|\Omega\|_{q}\right)}}\left|\int_{S^{n-1}} \Omega\left(y^{\prime}\right) G_{k+j}(f)\left(x-2^{k \log \left(e+\|\Omega\|_{q}\right)} r y^{\prime}\right) d \sigma\left(y^{\prime}\right)\right|^{2} r^{-1} d r\right)^{1 / 2} .
$$

Then

$$
M_{\Omega}(f)(x) \leq \sum_{j \in \mathbb{Z}} E_{j}(f)(x)
$$

By exactly the same argument in [2], we obtain

$$
\left\|E_{j}(f)\right\|_{2} \leq C 2^{-\beta|j| / q^{\prime}} \log ^{1 / 2}\left(e+\|\Omega\|_{q}\right)\|f\|_{2} .
$$

On the other hand, by a duality argument; see (3.24)-(3.25) for similar argument, we get

$$
\left\|E_{j}(f)\right\|_{p} \leq C \log ^{1 / 2}\left(e+\|\Omega\|_{q}\right)\|f\|_{p}
$$

for all $2<p<\infty$. Thus, by interpolation between (2.17) and (2.18), we have

$$
\left\|E_{j}(f)\right\|_{p} \leq C 2^{-\varepsilon\left(|j| / q^{\prime}\right)} \log ^{1 / 2}\left(e+\|\Omega\|_{q}\right)\|f\|_{p}
$$


for some $\varepsilon>0$ and for all $2 \leq p<\infty$, and $j \in \mathbb{Z}$ with constant $C$ independent of $\Omega, k$, and $j$. Hence, (2.14) follows by (2.16) and (2.19). This completes the proof.

\section{Proof of Theorem 1.1}

Proof of Theorem 1.1. We will argue by induction on the degree of the polynomial $P$. If $d=\operatorname{deg}(P)=0$, then (1.4) follows easily from Lemma 2.4. In fact, if $d=0$, then by duality it can be easily seen that

$$
M_{\Omega, P}(f)(x) \leq C M_{\Omega}(f)(x)
$$

Thus, by Lemma 2.4, we have

$$
\begin{aligned}
\left\|M_{\Omega, P}(f)\right\|_{p} & \leq\left\{\frac{2^{1 / q^{\prime}}}{2^{1 / q^{\prime}}-1}\right\} \log ^{1 / 2}\left(e+\|\Omega\|_{q}\right) C_{p}\|f\|_{p} \\
& \leq\left\{\frac{2^{1 / q^{\prime}}}{2^{1 / q^{\prime}}-1}\right\}\left\{1+\log ^{1 / 2}\left(e+\|\Omega\|_{q}\right)\right\} C_{p}\|f\|_{p}
\end{aligned}
$$

for all $p \geq 2$.

Now, if $d=1$, that is, $P(y)=\vec{a} \cdot y$ for some $\vec{a} \in \mathbb{R}^{n}$, then by (3.2), we have

$$
\begin{aligned}
\left\|M_{\Omega, P}(f)\right\|_{p} & \leq\left\{\frac{2^{1 / q^{\prime}}}{2^{1 / q^{\prime}}-1}\right\}\left\{\log ^{1 / 2}\|\Omega\|_{q}\right\} C_{p}\|g\|_{p} \\
& =\left\{\frac{2^{1 / q^{\prime}}}{2^{1 / q^{\prime}}-1}\right\}\left\{1+\log ^{1 / 2}\left(e+\|\Omega\|_{q}\right)\right\} C_{p}\|f\|_{p},
\end{aligned}
$$

where $g(y)=e^{-i P(y)} f(y)$.

Next, assume that (1.4) holds for all polynomials $Q$ of degree less than or equal to $d>1$. Let

$$
P(x)=\sum_{|\alpha| \leq d+1} a_{\alpha} x^{\alpha}
$$

be a polynomial of degree $d+1$. Then by duality, we have

$$
M_{\Omega, P}(f)(x)=\left(\int_{0}^{\infty}\left|\int_{S^{n-1}} e^{i P\left(r y^{\prime}\right)} \Omega\left(y^{\prime}\right) f\left(x-r y^{\prime}\right) d \sigma\left(y^{\prime}\right)\right|^{2} r^{-1} d r\right)^{1 / 2}
$$

We may assume that $P$ does not contain $|x|^{d+1}$ as one of its terms. By dilation invariance, we may also assume that

$$
\sum_{|\alpha|=d+1}\left|a_{\alpha}\right|=1
$$


8 A unifying approach for certain class of maximal functions

We now choose a collection $\left\{\omega_{k}\right\}_{k \in \mathbb{Z}}$ of $\mathscr{C}^{\infty}$ functions defined on $(0, \infty)$ that satisfy the following properties:

$$
\begin{gathered}
\operatorname{supp}\left(\psi_{k}\right) \subseteq\left[2^{-\log \left(e+\|\Omega\|_{q}\right)(k+1)}, 2^{-\log \left(e+\|\Omega\|_{q}\right)(k-1)}\right], \quad 0 \leq \psi_{k} \leq 1, \\
\sum_{k \in \mathbb{Z}} \psi_{k}(u)=1 .
\end{gathered}
$$

Set

$$
\eta_{\infty}(u)=\sum_{k=-\infty}^{0} \psi_{k}(u), \quad \eta_{0}(u)=\sum_{k=1}^{\infty} \psi_{k}(u) .
$$

Then,

$$
\begin{gathered}
\eta_{\infty}(u)+\eta_{0}(u)=1, \\
\operatorname{supp}\left(\eta_{\infty}(u)\right) \subset\left[2^{-\log \left(e+\|\Omega\|_{q}\right)}, \infty\right), \quad \operatorname{supp}\left(\eta_{0}(u)\right) \subset(0,1] .
\end{gathered}
$$

Define the operators $\mathscr{S}_{\Omega, P, \infty}$ and $\mathscr{Y}_{\Omega, P, 0}$ by

$$
\begin{gathered}
\mathscr{S}_{\Omega, P, \infty}(f)(x)=\left(\int_{2^{-\log (e+\mid\|\Omega\| q)}}^{\infty}\left|\eta_{\infty}(r) \int_{S^{n-1}} e^{i P\left(r y^{\prime}\right)} \Omega\left(y^{\prime}\right) f\left(x-r y^{\prime}\right) d \sigma\left(y^{\prime}\right)\right|^{2} r^{-1} d r\right)^{1 / 2}, \\
\mathscr{I}_{\Omega, P, 0}(f)(x)=\left(\int_{0}^{1}\left|\eta_{0}(r) \int_{S^{n-1}} e^{i P\left(r y^{\prime}\right)} \Omega\left(y^{\prime}\right) f\left(x-r y^{\prime}\right) d \sigma\left(y^{\prime}\right)\right|^{2} r^{-1} d r\right)^{1 / 2} .
\end{gathered}
$$

Thus, by (3.9), we have

$$
\mathscr{Y}_{\Omega, P}(f)(x) \leq \mathscr{S}_{\Omega, P, 0}(f)(x)+\mathscr{Y}_{\Omega, P, \infty}(f)(x) .
$$

Now, we estimate $\left\|\mathscr{I}_{\Omega, P, 0}\right\|_{p}$.

Let

$$
Q(x)=\sum_{|\alpha| \leq d} a_{\alpha} x^{\alpha}
$$

Assume that $\operatorname{deg}(Q)=l$, where $0 \leq l \leq d$. Define the operators $\mathscr{Y}_{\Omega, P, 0}^{(1)}$ and $\mathscr{Y}_{\Omega, Q, 0}^{(2)}$ by

$$
\begin{gathered}
\mathscr{S}_{\Omega, P, 0}^{(1)}(f)(x)=\left(\int_{0}^{1}\left|\int_{S^{n-1}}\left(e^{i P\left(r y^{\prime}\right)}-e^{i Q\left(r y^{\prime}\right)}\right) \Omega(y) f\left(x-r y^{\prime}\right) d \sigma\left(y^{\prime}\right)\right|^{2} r^{-1} d r\right)^{1 / 2}, \\
\mathscr{S}_{\Omega, Q, 0}^{(2)}(f)(x)=\left(\int_{0}^{1}\left|\int_{S^{n-1}} e^{i Q\left(r y^{\prime}\right)} \Omega\left(y^{\prime}\right) f\left(x-r y^{\prime}\right) d \sigma\left(y^{\prime}\right)\right|^{2} r^{-1} d r\right)^{1 / 2} .
\end{gathered}
$$

Now, by the observation that $\eta_{0}(r) \leq 1$ and by Minkowski's inequality, we obtain

$$
\mathscr{Y}_{\Omega, P, 0}(f)(x) \leq \mathscr{Y}_{\Omega, P, 0}^{(1)}(f)(x)+\mathscr{Y}_{\Omega, Q, 0}^{(2)}(f)(x) .
$$


By induction assumption, it follows that

$$
\left\|\mathscr{S}_{\Omega, Q, 0}^{(2)}(f)\right\|_{p} \leq\left\{1+\log ^{1 / 2}\left(e+\|\Omega\|_{q}\right)\right\}\left\{\frac{2^{1 / q^{\prime}}}{2^{1 / q^{\prime}}-1}\right\} C_{p}\|f\|_{p}
$$

for all $p \geq 2$.

On the other hand, by Cauchy-Schwarz inequality, by the fact that $\|\Omega\|_{1} \leq 1$, and the inequality

$$
\begin{aligned}
\left|\left(e^{i P\left(r y^{\prime}\right)}-e^{i Q\left(r y^{\prime}\right)}\right)\right| & \leq r^{d+1}\left|\sum_{|\alpha|=d+1} a_{\alpha} y^{\prime \alpha}\right| \\
& \leq r^{d+1},
\end{aligned}
$$

we get

$$
\begin{aligned}
\mathscr{S}_{\Omega, P, 0}^{(1)}(f)(x) & \leq\left(\int_{0}^{1} \int_{S^{n-1}}\left|\left(e^{i P\left(r y^{\prime}\right)}-e^{i Q\left(r y^{\prime}\right)}\right)\right|^{2}\left|\Omega\left(y^{\prime}\right)\right|\left|f\left(x-r y^{\prime}\right)\right|^{2} d \sigma\left(y^{\prime}\right) r^{-1} d r\right)^{1 / 2} \\
& \leq\left(\int_{0}^{1} \int_{S^{n-1}}\left|\Omega\left(y^{\prime}\right)\right|\left|f\left(x-r y^{\prime}\right)\right|^{2} d \sigma\left(y^{\prime}\right) r^{2 d+1} d r\right)^{1 / 2} \\
& =\left(\sum_{j=-\infty}^{-1} \int_{2^{j}}^{2^{j+1}} \int_{S^{n-1}}\left|\Omega\left(y^{\prime}\right)\right|\left|f\left(x-r y^{\prime}\right)\right|^{2} d \sigma(y) r^{2 d+1} d r\right)^{1 / 2} \\
& \leq\left(\sum_{j=-\infty}^{-1} 2^{(2 d+2) j} \int_{2^{j}}^{2^{j+1}} \int_{S^{n-1}}\left|\Omega\left(y^{\prime}\right)\right|\left|f\left(x-r y^{\prime}\right)\right|^{2} d \sigma(y) r^{-1} d r\right)^{1 / 2} \\
& \leq C\left(M_{\Omega}\left(|f|^{2}\right)\right)^{1 / 2}(x),
\end{aligned}
$$

where $M_{\Omega}$ is the operator given by (2.1) with $\mathscr{P}(y)=y$. Thus, by (3.17), by the fact that $\|\Omega\|_{1} \leq 1$, and Lemma 2.1, we obtain

$$
\left\|\mathscr{S}_{\Omega, P, 0}^{(1)}(f)\right\|_{p} \leq C_{p}\|f\|_{p}
$$

for all $p \geq 2$ with constant $C_{p}$ independent of the function $\Omega$ and the coefficients of the polynomial $P$. Therefore, by (3.14), by Minkowski's inequality, by (3.15), and (3.18), we obtain

$$
\left\|\mathscr{S}_{\Omega, P, 0}(f)\right\|_{p} \leq\left\{1+\log ^{1 / 2}\left(e+\|\Omega\|_{q}\right)\right\}\left\{\frac{2^{1 / q^{\prime}}}{2^{1 / q^{\prime}}-1}\right\} C_{p}\|f\|_{p}
$$

for all $p \geq 2$. 
10 A unifying approach for certain class of maximal functions

Finally, we prove the $L^{p}$ boundedness of $\mathscr{Y}_{\Omega, P, \infty}$. By generalized Minkowski's inequality, we can write $\mathscr{Y}_{\Omega, P, \infty}$ as

$$
\begin{aligned}
\mathscr{Y}_{\Omega, P, \infty}(f)(x) & =\left(\int_{2^{-\log (e+\|\Omega\| q)}}^{\infty}\left|\eta_{\infty}(r) \int_{\mathbf{S}^{n-1}} e^{i P\left(r y^{\prime}\right)} \Omega\left(y^{\prime}\right) f\left(x-r y^{\prime}\right) d \sigma\left(y^{\prime}\right)\right|^{2} r^{-1} d r\right)^{1 / 2} \\
& =\left(\int_{0}^{\infty}\left|\sum_{k=-\infty}^{0} \psi_{k}(r) \int_{S^{n-1}} e^{i P\left(r y^{\prime}\right)} \Omega\left(y^{\prime}\right) f\left(x-r y^{\prime}\right) d \sigma\left(y^{\prime}\right)\right|^{2} \frac{1}{r} d r\right)^{1 / 2} \\
& \leq \sum_{k=-\infty}^{0} \mathscr{S}_{\Omega, P, \infty, k}(f)(x),
\end{aligned}
$$

where

$$
\mathscr{Y}_{\Omega, P, \infty, k}(f)(x)=\left(\int_{2^{-\log (e+\|\Omega\| q)(k+1)}}^{2^{-\log (e+\mid\|\Omega\| q)(k-1)}}\left|\int_{S^{n-1}} e^{i P\left(r y^{\prime}\right)} \Omega\left(y^{\prime}\right) f\left(x-r y^{\prime}\right) d \sigma\left(y^{\prime}\right)\right|^{2} r^{-1} d r\right)^{1 / 2} .
$$

By Plancherel's theorem, Fubini's theorem, and Lemma 2.3, we have

$$
\left\|\mathscr{S}_{\Omega, P, \infty, k}(f)\right\|_{2}^{2}=\int_{\mathbb{R}^{n}}|\hat{f}(\xi)|^{2} \mathbf{J}_{k, \Omega}(\xi) d \xi \leq 2^{(k+1) / 4 q^{\prime}} \log \left(e+\|\Omega\|_{q}\right)\|f\|_{2}^{2}
$$

Thus,

$$
\left\|\mathscr{S}_{\Omega, P, \infty, k}(f)\right\|_{2} \leq 2^{(k+1) / 8 q^{\prime}} \log ^{1 / 2}\left(e+\|\Omega\|_{q}\right)\|f\|_{2} .
$$

Now, for $p>2$, choose $g \in L^{(p / 2)^{\prime}}$ with $\|g\|_{(p / 2)^{\prime}}=1$ such that

$$
\begin{aligned}
& \left\|\mathscr{S}_{\Omega, P, \infty, k}(f)\right\|_{p}^{2} \\
& \quad=\int_{\mathbb{R}^{n}} \int_{1}^{2^{2 \log \left(e+\|\Omega\|_{q}\right)}}\left|\int_{S^{n-1}} E_{k, \Omega}\left(r, P\left(y^{\prime}\right), 0\right) \Omega\left(y^{\prime}\right) f\left(x-2^{-\gamma_{k, \Omega}} r y^{\prime}\right) d \sigma\left(y^{\prime}\right)\right|^{2} r^{-1} d r|g(x)| d x \\
& \quad \leq \int_{\mathbb{R}^{n}}|f(z)|^{2} \int_{1}^{2^{2 \log \left(e+\|\Omega\|_{q}\right)}} \int_{S^{n-1}}\left|\Omega\left(y^{\prime}\right)\right|\left|g\left(z+2^{-\gamma_{k, \Omega}} r y^{\prime}\right)\right| \frac{d \sigma\left(y^{\prime}\right) d r}{r} d z \\
& \quad \leq C \log \left(e+\|\Omega\|_{q}\right)\|f\|_{p}^{2}\left\|M_{\Omega} \tilde{g}(z)\right\|_{(p / 2)^{\prime}},
\end{aligned}
$$

where $M_{\Omega}$ is the operator given by (2.1) with $\mathscr{P}(y)=y$. Thus, Lemma 2.1 and (3.24) imply that

$$
\left\|\mathscr{S}_{\Omega, P, \infty, k}(f)\right\|_{p} \leq \log ^{1 / 2}\left(e+\|\Omega\|_{q}\right) C\|f\|_{p},
$$

which when combined with (3.23) implies

$$
\left\|\mathscr{S}_{\Omega, P, \infty, k}(f)\right\|_{p} \leq 2^{(k+1) \delta / 8 q^{\prime}} \log ^{1 / 2}\left(e+\|\Omega\|_{q}\right) C\|f\|_{p},
$$


where $\delta$ is a constant that is independent of the essential variables. Thus, by (3.20), (3.26), and Minkowski's inequality, we get

$$
\left\|\mathscr{S}_{\Omega, P, \infty}(f)\right\|_{p} \leq C \log ^{1 / 2}\left(e+\|\Omega\|_{q}\right)\left\{\frac{2^{1 / q^{\prime}}}{2^{1 / q^{\prime}}-1}\right\} C_{p}\|f\|_{p}
$$

for all $p \geq 2$. Hence, by Minkowski's inequality, (3.11), (3.19), and (3.27), we obtain (1.4) for the given polynomial $P$. This completes the proof.

\section{Proof of results concerning $L(\log L)^{1 / 2}\left(\mathbf{S}^{n-1}\right)$}

Proof of Theorem 1.2. Given $\Omega \in L(\log L)^{1 / 2}\left(\mathbf{S}^{n-1}\right)$, then we decompose $\Omega$ as a sum of functions in $L^{2}\left(\mathbf{S}^{n-1}\right)$. More precisely, there exists a sequence $\left\{\Omega_{m}: m=0,1,2, \ldots\right\}$ of functions in $L^{1}\left(\mathbf{S}^{n-1}\right)$ with

$$
\Omega=\sum_{m=0}^{\infty} \Omega_{m}
$$

such that

$$
\begin{gathered}
\int_{\mathbf{S}^{n-1}} \Omega_{m}\left(y^{\prime}\right) d \sigma\left(y^{\prime}\right)=0, \quad\left\|\Omega_{m}\right\|_{1} \leq C, \quad \Omega_{0} \in L^{2}\left(\mathbf{S}^{n-1}\right), \\
\left\|\Omega_{m}\right\|_{\infty} \leq 2^{4 m} C \quad \text { for } m=1,2,3, \ldots, \\
\sum_{m=1}^{\infty} \sqrt{m}\left\|\Omega_{m}\right\|_{1} \leq\|\Omega\|_{L(\log L)^{1 / 2}\left(\mathbf{S}^{n-1}\right)} C .
\end{gathered}
$$

For a detailed proof of the existence of the decomposition (4.1), one might look into $[2,5]$.

Now, by (4.1), we have the following:

$$
M_{\Omega, P}(f)(x) \leq M_{\Omega_{0}, P}(f)(x)+\sum_{m=1}^{\infty}\left\|\Omega_{m}\right\|_{1} M_{\Omega_{m}, P}(f)(x) .
$$

By Lemma 2.4, we have

$$
\left\|M_{\Omega_{0}, P}(f)\right\|_{p} \leq\left\{1+\log ^{1 / 2}\left(e+\left\|\Omega_{0}\right\|_{2}\right)\right\} C_{p}\|f\|_{p}
$$

for all $p \geq 2$.

Next, by observing that

$$
1+\log ^{1 / 2}\left(e+\left\|\Omega_{m}\right\|_{\infty}\right) \leq 1+\log ^{1 / 2}\left(e+2^{4 m}\right) \leq 4 \sqrt{m}
$$

for all $m \geq 1$, Theorem 1.1 implies that

$$
\left\|M_{\Omega_{m}, P}(f)\right\|_{p} \leq 4 \sqrt{m} C_{p}\|f\|_{p}
$$

for all $p \geq 2$ with constant $C_{p}$ independent of $m$. 
Thus, by Minkowski’s inequality, (4.4), (4.5), (4.7), and (4.2), we obtain

$$
\left\|M_{\Omega, P}(f)\right\|_{p} \leq C_{p}\|f\|_{p}
$$

for all $p \geq 2$. This completes the proof.

Proof of Corollary 1.5. By the inequality (1.5) and the decomposition (4.1), we have

$$
\left|T_{\Omega, P, h}(f)(x)\right| \leq\left|T_{\Omega_{0}, P, h}(f)(x)\right|+\sum_{m=1}^{\infty}\left\|\Omega_{m}\right\|_{1}\left|T_{\Omega_{m}, P, h}(f)(x)\right| .
$$

Thus, by Theorem 1.4, (4.9), and a similar argument as in the proof of Theorem 1.2, the proof is complete.

\section{Proof of results concerning block spaces}

We start this section by recalling the definition of block spaces introduced by Jiang and Lu (see [16]).

Definition 5.1. (1) For $x_{0}^{\prime} \in \mathbf{S}^{n-1}$ and $0<\theta_{0} \leq 2$, the set $B\left(x_{0}^{\prime}, \theta_{0}\right)=\left\{x^{\prime} \in \mathbf{S}^{n-1}:\left|x^{\prime}-x_{0}^{\prime}\right|<\right.$ $\left.\theta_{0}\right\}$ is called a cap on $\mathbf{S}^{n-1}$.

(2) For $1<q \leq \infty$, a measurable function $b$ is called a $q$-block on $\mathbf{S}^{n-1}$ if $b$ is a function supported on some cap $I=B\left(x_{0}^{\prime}, \theta_{0}\right)$ with $\|b\|_{L^{q}} \leq|I|^{-1 / q^{\prime}}$, where $|I|=\sigma(I)$ and $1 / q+$ $1 / q^{\prime}=1$.

(3) $B_{q}^{\kappa, v}\left(\mathbf{S}^{n-1}\right)=\left\{\Omega \in L^{1}\left(\mathbf{S}^{n-1}\right): \Omega=\sum_{\mu=1}^{\infty} c_{\mu} b_{\mu}\right.$, where each $c_{\mu}$ is a complex number; each $b_{\mu}$ is a $q$-block supported on a cap $I_{\mu}$ on $S^{n-1}$; and $M_{q}^{\kappa, v}\left(\left\{c_{\mu}\right\},\left\{I_{\mu}\right\}\right)=\sum_{\mu=1}^{\infty}\left|c_{\mu}\right|(1+$ $\left.\phi_{\kappa, v}\left(\left|I_{\mu}\right|\right)\right)<\infty$, where $\phi_{\kappa, v}(t)=\int_{t}^{1} u^{-1-\kappa} \log ^{v}\left(u^{-1}\right) d u$ if $0<t<1$ and $\phi_{\kappa, v}(t)=0$ if $\left.t \geq 1\right\}$.

Notice that $\phi_{\kappa, v}(t) \sim t^{-\kappa} \log ^{v}\left(t^{-1}\right)$ as $t \rightarrow 0$ for $\kappa>0, v \in \mathbb{R}$, and $\phi_{0, v}(t) \sim \log ^{v+1}\left(t^{-1}\right)$ as $t \rightarrow 0$ for $v>-1$. Moreover, among many properties of block spaces [17], we cite the following which are closely related to our work:

$$
\begin{aligned}
B_{q}^{0,0} & \subset B_{q}^{0,-1 / 2} \quad(q>1), \\
B_{q_{2}}^{0, v} & \subset B_{q_{1}}^{0, v} \quad\left(1<q_{1}<q_{2}\right), \\
L^{q}\left(\mathbf{S}^{n-1}\right) & \subseteq B_{q}^{0, v}\left(\mathbf{S}^{n-1}\right) \quad(\text { for } v>-1), \\
\bigcup_{q>1} B_{q}^{0, v}\left(\mathbf{S}^{n-1}\right) & \nsubseteq \bigcup_{p>1} L^{p}\left(\mathbf{S}^{n-1}\right) \quad \text { for any } v>-1 .
\end{aligned}
$$

Proof of Theorem 1.3. Assume that $\Omega \in B_{q}^{0,-1 / 2}\left(\mathbf{S}^{n-1}\right), q>1$. Then

$$
\Omega=\sum_{\mu=1}^{\infty} c_{\mu} b_{\mu}
$$


where each $c_{\mu}$ is a complex number; each $b_{\mu}$ is a $q$-block supported on a cap $I_{\mu}$ on $\mathbf{S}^{n-1}$; and

$$
M_{q}^{0,-1 / 2}\left(\left\{c_{\mu}\right\},\left\{I_{\mu}\right\}\right)=\sum_{\mu=1}^{\infty}\left|c_{\mu}\right|\left(1+\log ^{1 / 2}\left(\left|I_{\mu}\right|^{-1}\right)\right)<\infty .
$$

Without loss of generality, we may assume that $\left|I_{\mu}\right|<1$. For each $\mu$, let

$$
\bar{b}_{\mu}(x)=b_{\mu}(x)-\int_{S^{n-1}} b_{\mu}(u) d u .
$$

Then, it follows that

$$
\left\|\bar{b}_{\mu}\right\|_{q} \leq C|I|^{-1 / q^{\prime}}, \quad\left\|\bar{b}_{\mu}\right\|_{1} \leq C .
$$

By the decomposition (5.3), we have

$$
M_{\Omega, P}(f)(x) \leq \sum_{\mu=1}^{\infty}\left|c_{\mu}\right| M_{\bar{b}_{\mu}, P}(f)(x) .
$$

Thus, by Minkowski's inequality, (5.5), and Theorem 1.1, we have

$$
\begin{aligned}
\left\|\mathcal{M}_{\Omega, P}(f)\right\|_{p} & \leq C_{p} \sum_{\mu=1}^{\infty}\left|c_{\mu}\right|\left\{1+\log ^{1 / 2}\left(e+|I|^{-1 / q^{\prime}}\right)\right\}\|f\|_{p} \\
& \leq C_{p, q} \sum_{\mu=1}^{\infty}\left|c_{\mu}\right|\left(1+\log ^{1 / 2}\left(\left|I_{\mu}\right|^{-1}\right)\right)\|f\|_{p} \\
& \leq \widetilde{C}_{p, q}\|f\|_{p}
\end{aligned}
$$

for all $p \geq 2$, where the last inequality follows by (5.3). This completes the proof.

A proof of Corollary 1.6 can be obtained by a similar argument as in the proof of Corollary 1.5. We omit the details.

\section{Further applications}

This section is devoted to present some results that follow by applying our results in Section 1.

Parametric Marcinkiewicz integral operators. The parametric Marcinkiewicz integral operator related to the operator $M_{\Omega, P}$ is defined by

$$
\mu_{\Omega, P}^{\rho} f(x)=\left(\left.\left.\int_{-\infty}^{\infty}\left|2^{-\rho t} \int_{|y| \leq 2^{t}} e^{i P(y)} f(x-y)\right| y\right|^{-n+\rho} \Omega(y) d y\right|^{2} d t\right)^{1 / 2},
$$

where $\rho$ is a positive real number. Clearly, when $P=0$, the operator $\mu_{\Omega}^{\rho}=\mu_{\Omega, 0}^{\rho}$ is the wellknown parametric Marcinkiewicz integral operator introduced by Hörmander [15]. 
Now, it is straightforward to see that

$$
\mu_{\Omega, P}^{\rho} f(x) \leq C(\rho) M_{\Omega, P} f(x)
$$

Therefore, by (6.2), Theorem 1.1, and the decompositions (4.1) and (5.2), we can easily obtain the following theorem.

Theorem 6.1. Suppose that $\rho>0$ and that $\Omega \in L(\log L)^{1 / 2}\left(\mathbf{S}^{n-1}\right)$ satisfying (1.1). Then the parametric Marcinkiewicz integral operator $\mu_{\Omega, P}^{\rho}$ is bounded on $L^{p}$ for all $p \geq 2$ with $L^{p}$ bounds that may depend on the degree of the polynomial $P$ but they are independent of the function $\Omega$ and the coefficients of the polynomial $P$.

Theorem 6.2. Suppose that $\rho>0$ and that $\Omega \in B_{q}^{0,-1 / 2}\left(\mathbf{S}^{n-1}\right), q>1$, satisfying (1.1). Then the parametric Marcinkiewicz integral operator $\mu_{\Omega, P}^{\rho}$ is bounded on $L^{p}$ for all $p \geq 2$ with $L^{p}$ bounds that may depend on the degree of the polynomial $P$ but they are independent of the function $\Omega$ and the coefficients of the polynomial $P$.

We remark here that by specializing to the case $P=0$ and $\rho=1$, the resulting operator $\mu_{\Omega}=\mu_{\Omega, 0}^{1}$ is the classical Marcinkiewicz integral operator introduced by Stein [25]. Thus, Theorems 6.1 and 6.2 generalize as well as improve the result in (see [25]). Furthermore, Theorems 6.1 and 6.2 generalize the corresponding results in [2, 3, 8]. For more background information and related results about Marcinkiewicz integral operators, we refer the readers to consult $[6,8,15,25]$, and the references therein.

Morrey spaces. In [20], Mizuhara introduced the following generalized Morrey spaces.

Definition 6.3. Let $\phi:(0, \infty) \rightarrow(0, \infty)$ be an increasing function that satisfies $\phi(2 r) \leq$ $D \phi(r)$ for any $r>0$, where $D$ is a constant independent of $r$. Let $1 \leq p<\infty$. A locally integrable function $f \in L^{p, \phi}$ if

$$
\int_{B_{r}\left(x_{0}\right)}|f(x)|^{p} d x \leq C^{p} \phi(r)
$$

for all $x_{0} \in \mathbb{R}^{n}$ and $r>0$, where $B_{r}\left(x_{0}\right)$ is the ball with center $x_{0}$ and radius $r$.

It is worth pointing out here that Morrey spaces have been used to study several problems in harmonic analysis, such as studying the local behavior of solutions to secondorder elliptic partial differential equations and measuring the regularity of the solution to an elliptic second-order equation with discontinuous coefficients; see $[13,21]$, and references therein.

By Theorem 1.1, the decompositions (4.1) and (5.2), and following a similar argument as in the proof of Theorem 5 in [13], we obtain the following theorem.

Theorem 6.4. Suppose that $\Omega \in L\left(\log ^{+} L\right)^{1 / 2}\left(\mathbf{S}^{n-1}\right) \cup B_{q}^{0,-1 / 2}\left(\mathbf{S}^{n-1}\right), q>1$, satisfying (1.1). Let $P: \mathbb{R}^{n} \rightarrow \mathbb{R}$ be a real-valued polynomial. Then $M_{\Omega, P}$ is bounded on $L^{p, \phi}\left(\mathbb{R}^{n}\right)$ for all $p \geq 2$ with $L^{p}$ bounds independent of the coefficients of the polynomial $P$.

Hence, by (6.2) and Theorem 6.4, we obtain that the operator $\mu_{\Omega, P}^{\rho}$ is bounded on $L^{p, \phi}\left(\mathbb{R}^{n}\right)$ for all $p \geq 2$ with $L^{p}$ bounds independent of the coefficients of the polynomial 
$P$. Moreover, by (1.5) and Theorem 6.4, it follows that the operator $T_{\Omega, P, h}$ is bounded on $L^{p, \phi}$ for all $1<p<\infty$ and $h \in L^{2}\left(\mathbb{R}_{+}, r^{-1} d r\right)$.

$L^{p}$ estimates with radial weights. The results in this paper can be easily extended to the radial weights setting introduced by Duoandikoetxea [9]. In order to state our weighted $L^{p}$ estimates, we recall the definition of the radial weights $[9,13]$.

Definition 6.5. Suppose that $\omega(t) \geq 0$ and $\omega \in L_{\text {loc }}^{1}\left(\mathbb{R}^{+}\right)$. For $1<p<\infty, \omega \in A_{p}\left(\mathbb{R}^{+}\right)$if there is a constant $C>0$ such that for any interval $I \subseteq \mathbb{R}^{+}$,

$$
\left(|I|^{-1} \int_{I} \omega(t) d t\right)\left(|I|^{-1} \int_{I} \omega(t)^{-1 /(p-1)} d t\right) \leq C<\infty
$$

If there is a constant $C>0$ such that

$$
\omega^{*}(t) \leq C \omega(t) \text { for a.e. } t \in \mathbb{R}^{+},
$$

where $\omega^{*}$ is the Hardy-Littlewood maximal function of $\omega$ on $\mathbb{R}^{+}$, then $\omega \in A_{1}\left(\mathbb{R}^{+}\right)$.

We let $\widetilde{A}_{p}\left(\mathbb{R}^{+}\right)$be the class of functions $\omega$ that can be written as follows: $\omega(x)=$ $\nu_{1}(|x|) \nu_{2}(|x|)^{1-p}$, where either $v_{i} \in A_{1}\left(\mathbb{R}^{+}\right)$is decreasing or $v_{i}^{2} \in A_{1}\left(\mathbb{R}^{+}\right), i=1,2$. Also, for $1<p<\infty$, we let

$$
\bar{A}_{p}\left(\mathbb{R}^{+}\right)=\left\{\omega(x)=\omega(|x|): \omega(t)>0, \omega(t) \in L_{\mathrm{loc}}^{1}\left(\mathbb{R}^{+}\right), \omega^{2}(t) \in A_{p}\left(\mathbb{R}^{+}\right)\right\}
$$

and let $A_{p}^{I}\left(\mathbb{R}^{n}\right)$ be the weighted class defined by using all $n$-dimensional intervals with sides parallel to coordinate axes. The weighted $L^{p}$ space $L^{p}\left(\mathbb{R}^{n}, \omega(x) d x\right)$ associated to the weight $\omega$ is defined to be the class of all measurable functions $f$ with $\|f\|_{L^{p}(\omega)}<\infty$, where

$$
\|f\|_{L^{p}(\omega)}=\left(\int_{\mathbb{R}^{n}}|f(x)|^{p} \omega(x) d x\right)^{1 / p} .
$$

It is known that $\bar{A}_{p}\left(\mathbb{R}^{+}\right) \subseteq \widetilde{A}_{p}\left(\mathbb{R}^{+}\right)$; see [13]. Moreover, if $\omega(t) \in \bar{A}_{p}\left(\mathbb{R}^{+}\right)$, then $\omega(|x|)$ is in Muckenhoupt weighted class $A_{p}\left(\mathbb{R}^{n}\right)$ whose definition can be found in [14].

By the same argument in this paper with minor modifications, it can be easily shown that the weighted version of all $L^{p}$ estimates obtained in this paper holds. In particular, we have the following theorem.

Theorem 6.6. Suppose that $\rho>0$ and that $\Omega \in L\left(\log ^{+} L\right)^{1 / 2}\left(\mathbf{S}^{n-1}\right) \cup B_{q}^{0,-1 / 2}\left(\mathbf{S}^{n-1}\right), q>1$, satisfying (1.1). Let $P: \mathbb{R}^{n} \rightarrow \mathbb{R}$ be a real-valued polynomial. If $\omega \in \tilde{A}_{p / 2} \cap A_{p / 2}^{I}, 2 \leq p<\infty$, then the operators $M_{\Omega, P}$ and $\mu_{\Omega, P}^{\rho}$ are bounded on $L^{p}(\omega)$ with $L^{p}$ bounds independent of the coefficients of the polynomial $P$.

A special class of radial weights that have received a considerable amount of attention is the class of power weights $|x|^{\alpha}$. For background information and related results on power weights, we refer the readers to consult $[9,13]$, among others. By the observation that $|x|^{\alpha} \in \tilde{A}_{p / 2} \cap A_{p / 2}^{I}$ if $\alpha \in(-1, p / 2-1)$, it follows from Theorem 6.6 that the following holds. 
Corollary 6.7. Suppose that $\rho>0$ and that $\Omega \in L\left(\log ^{+} L\right)^{1 / 2}\left(\mathbf{S}^{n-1}\right) \cup B_{q}^{0,-1 / 2}\left(\mathbf{S}^{n-1}\right), q>1$, satisfying (1.1). Let $P: \mathbb{R}^{n} \rightarrow \mathbb{R}$ be a real-valued polynomial. Then the operators $M_{\Omega, P}$ and $\mu_{\Omega, P}^{\rho}$ are bounded on $L^{p}\left(|x|^{\alpha}\right)$ if $\alpha \in(-1, p / 2-1)$ with $L^{p}\left(|x|^{\alpha}\right)$ bounds independent of the coefficients of the polynomial $P$.

\section{Acknowledgment}

The author wishs to thank the referee of this paper for helpful comments.

\section{References}

[1] A. Al-Salman, Rough oscillatory singular integral operators of nonconvolution type, Journal of Mathematical Analysis and Applications 299 (2004), no. 1, 72-88.

[2] - On maximal functions with rough kernels in $L(\log L)^{1 / 2}\left(\mathbf{S}^{n-1}\right)$, Collectanea Mathematica 56 (2005), no. 1, 47-56.

[3] _ On a class of singular integral operators with rough kernels, Canadian Mathematical Bulletin 49 (2006), no. 1, 3-10.

[4] A. Al-Salman and A. Al-Jarrah, Rough oscillatory singular integral operators. II, Turkish Journal of Mathematics 27 (2003), no. 4, 565-579.

[5] A. Al-Salman and Y. Pan, Singular integrals with rough kernels in $L \log ^{+} L\left(\mathbf{S}^{n-1}\right)$, Journal of the London Mathematical Society. Second Series 66 (2002), no. 1, 153-174.

[6] J. Chen, D. S. Fan, and Y. Pan, A note on a Marcinkiewicz integral operator, Mathematische Nachrichten 227 (2001), no. 1, 33-42.

[7] L.-K. Chen and H. Lin, A maximal operator related to a class of singular integrals, Illinois Journal of Mathematics 34 (1990), no. 1, 120-126.

[8] Y. Ding, S. Lu, and K. Yabuta, A problem on rough parametric Marcinkiewicz functions, Journal of the Australian Mathematical Society 72 (2002), no. 1, 13-21.

[9] J. Duoandikoetxea, Weighted norm inequalities for homogeneous singular integrals, Transactions of the American Mathematical Society 336 (1993), no. 2, 869-880.

[10] D. S. Fan, K. Guo, and Y. Pan, $L^{p}$ estimates for singular integrals associated to homogeneous surfaces, Journal für die reine und angewandte Mathematik 542 (2002), 1-22.

[11] D. S. Fan and Y. Pan, Boundedness of certain oscillatory singular integrals, Studia Mathematica 114 (1995), no. 2, 105-116.

[12] Singular integral operators with rough kernels supported by subvarieties, American Journal of Mathematics 119 (1997), no. 4, 799-839.

[13] D. S. Fan, Y. Pan, and D. Yang, A weighted norm inequality for rough singular integrals, The Tohoku Mathematical Journal. Second Series 51 (1999), no. 2, 141-161.

[14] J. García-Cuerva and J. L. Rubio de Francia, Weighted Norm Inequalities and Related Topics, North-Holland Mathematics Studies, vol. 116, North-Holland, Amsterdam, 1985.

[15] L. Hörmander, Estimates for translation invariant operators in $L^{p}$ spaces, Acta Mathematica 104 (1960), 93-140.

[16] Y. S. Jiang and S. Z. Lu, Oscillatory singular integrals with rough kernel, Harmonic Analysis in China (M. D. Cheng, D. G. Deng, S. Gong, and C.-C. Yang, eds.), Math. Appl., vol. 327, Kluwer Academic, Dordrecht, 1995, pp. 135-145.

[17] M. Keitoku and E. Sato, Block spaces on the unit sphere in $\mathbf{R}^{n}$, Proceedings of the American Mathematical Society 119 (1993), no. 2, 453-455. 
[18] S. Lu, M. Taibleson, and G. Weiss, Spaces Generated by Blocks, Beijing Normal University Press, Beijing, 1989.

[19] S. Z. Lu and Y. Zhang, Criterion on $L^{p}$-boundedness for a class of oscillatory singular integrals with rough kernels, Revista Matemática Iberoamericana 8 (1992), no. 2, 201-219.

[20] T. Mizuhara, Boundedness of some classical operators on generalized Morrey spaces, Harmonic Analysis (Sendai, 1990) (S. Igari, ed.), ICM-90 Satell. Conference Proceedings, Springer, Tokyo, 1991, pp. 183-189.

[21] C. B. Morrey Jr., On the solutions of quasi-linear elliptic partial differential equations, Transactions of the American Mathematical Society 43 (1938), no. 1, 126-166.

[22] Y. Pan, $L^{2}$ estimates for convolution operators with oscillating kernels, Mathematical Proceedings of the Cambridge Philosophical Society 113 (1993), no. 1, 179-193.

[23] F. Ricci and E. M. Stein, Harmonic analysis on nilpotent groups and singular integrals. I. Oscillatory integrals, Journal of Functional Analysis 73 (1987), no. 1, 179-194.

[24] P. Sjölin, Convolution with oscillating kernels, Indiana University Mathematics Journal 30 (1981), no. $1,47-55$.

[25] E. M. Stein, On the functions of Littlewood-Paley, Lusin, and Marcinkiewicz, Transactions of the American Mathematical Society 88 (1958), no. 2, 430-466.

[26] _ Harmonic Analysis: Real-Variable Methods, Orthogonality, and Oscillatory Integrals, Princeton Mathematical Series, vol. 43, Princeton University Press, New Jersey, 1993.

[27] S. Yano, Notes on Fourier analysis. XXIX. An extrapolation theorem, Journal of the Mathematical Society of Japan 3 (1951), 296-305.

Ahmad Al-Salman: Department of Mathematics, Faculty of Science, Yarmouk University, Irbid, Jordan

E-mail address: alsalman@yu.edu.jo 